\title{
Risk-Sensitive Particle-Filtering-based Prognosis Framework for Estimation of Remaining Useful Life in Energy Storage Devices
}

\author{
Marcos E. Orchard ${ }^{1}$, Liang Tang ${ }^{2}$, Bhaskar Saha ${ }^{3}$, Kai Goebel ${ }^{4}$, George Vachtsevanos ${ }^{2,5}$ \\ ${ }^{1}$ Electrical Engineering Department, Universidad de Chile, \\ Santiago 8370451, Chile \\ morchard@ing.uchile.cl \\ 2 Impact Technologies, LLC, Rochester, NY 14623, USA \\ liang.tang@impact-tek.com \\ ${ }^{3} \mathrm{MCT}$, Inc at NASA Ames Research Center, \\ MS 269-4, Moffett Field, CA. 94035, USA \\ bhaskar.saha@nasa.gov \\ ${ }^{4}$ NASA Ames Research Center, \\ MS 269-4, Moffett Field, CA. 94035, USA \\ kai.goebel@nasa.gov \\ ${ }^{5}$ School of Electrical and Computer Engineering, Georgia Institute of Technology, \\ Atlanta, GA 30332, USA \\ gjv@ece.gatech.edu
}

\begin{abstract}
Failure prognosis, and particularly representation and management of uncertainty in long-term predictions, is a topic of paramount importance not only to improve productivity and efficiency, but also to ensure safety in the system's operation. The use of particle filter (PF) algorithms - in combination with outer feedback correction loops has contributed significantly to the development of a robust framework for online estimation of the remaining useful equipment life. This paper explores the advantages and disadvantages of a Risk-Sensitive PF (RSPF) prognosis framework that complements the benefits of the classic approach, by representing the probability of rare events and highly non-monotonic phenomena within the formulation of the nonlinear dynamic equation that describes the evolution of the fault condition in time. The performance of this approach is thoroughly compared using a set of ad-hoc metrics. Actual data illustrating aging of an energy storage device (specifically battery capacity measurements [A-hr]) are used to test the proposed framework.
\end{abstract}

Keywords: Risk-sensitive particle filtering, failure prognosis, nonlinear state estimation, battery prognosis.

\section{Introduction}

A number of approaches have been suggested in the recent years for uncertainty representation and management in prediction. Probabilistic, soft computing methods, and tools derived from evidential theory or Dempster-Shafer theory 1 have been explored for this purpose. Although probabilistic methods offer a mathematically rigorous methodology, they typically require a statistically sufficient database to estimate the required distributions. Soft-computing methods (fuzzy logic) offer an alternative when scarce data or contradictory data are available. Dempster's rule of combination and similar concepts from evidential theory such as belief or plausibility (upper and lower bounds of probability) based on mass function calculations can support uncertainty representation and management tasks. Confidence Prediction Neural Networks (NN) 2 have also been used to represent and manage uncertainty using Parzen windows as the kernel and a structure based on Specht's General Regression NN 3. For tuning of model hyper-parameters given observations, probabilistic reliability analysis tools employing an inner-outer loop Bayesian update scheme 4 have been employed.

Particle-filtering (PF) based prognostic algorithms 5-12 have been established as the de facto state of the art in failure prognosis. $\mathrm{PF}$ algorithms allow to avoid the assumption of Gaussian (or log-normal) probability density function (pdf) in nonlinear processes, with unknown model parameters, and simultaneously help to consider non-uniform 
probabilities of failure for particular regions of the state domain. Particularly, the authors in 6 have proposed a mathematically rigorous method (based on PF, function kernels, and outer correction loops) to represent and manage uncertainty in long-term predictions. However, there are still unsolved issues regarding the proper representation for the probability of rare events and highly nonmonotonic phenomena, since these events are associated to particles located at the tails of the predicted probability density functions.

This paper presents a solution for this problem. The paper is structured as follows: Section 2 introduces the basics of particle filtering (PF) and its application to the field of failure prognostics. Section 3 presents the proposed Risk-Sensitive PF (RSPF) framework and analyses the main advantages and disadvantages of its implementation, using actual failure data measuring battery capacity ([A-hr]). Section 4 utilizes performance metrics to assess prognostic results and evaluates the RSPF, when compared to the classic PF prognosis framework 5-10. Section 5 states conclusions.

\section{Particle Filtering and Failure Prognosis}

\subsection{Risk-sensitive particle filtering}

Nonlinear filtering is defined as the process of using noisy observation data to estimate at least the first two moments of a state vector governed by a dynamic nonlinear, nonGaussian state-space model. From a Bayesian standpoint, a nonlinear filtering procedure intends to generate an estimate of the posterior probability density function $p\left(x_{t} \mid y_{1: t}\right)$ for the state, based on the set of received measurements. Particle Filtering $(\mathrm{PF})$ is an algorithm that intends to solve this estimation problem by efficiently selecting a set of $N$ particles $\left\{x^{(i)}\right\}_{i=1 \cdots N}$ and weights $\left\{w_{t}^{(i)}\right\}_{i=1 \cdots N}$, such that the state pdf may be approximated $13-16$ by

$$
\begin{aligned}
& \tilde{\pi}_{t}^{N}\left(x_{t}\right)=\sum_{i=1}^{N} w_{t}^{(i)} \delta\left(x_{t}-x_{t}^{(i)}\right) \\
& w\left(x_{0: t}\right)=\frac{\pi_{t}\left(x_{0: t}\right)}{q_{t}\left(x_{0: t}\right)} \propto \frac{p\left(y_{t} \mid x_{t}\right) p\left(x_{t} \mid x_{0: t-1}\right)}{q_{t}\left(x_{t} \mid x_{0: t-1}\right)}
\end{aligned}
$$

where $q_{t}\left(x_{0: t}\right)$ is referred to as the importance sampling density function 13 . The choice of this importance density function is critical for the performance of the particle filter scheme. In the particular case of nonlinear state estimation, the value of the particle weights $w_{0: t}^{(i)}$ is computed by setting the importance density function equal to the a priori pdf for the state, i.e., $q_{t}\left(x_{0: t} \mid x_{0: t-1}\right)=p\left(x_{t} \mid x_{t-1}\right) 14$. Although this choice of importance density is appropriate for estimating the most likely probability distribution according to a particular set of measurement data, it does not offer a good estimate of the probability of events associated to high-risk conditions with low likelihood.

In contrast, the risk-sensitive particle filter (RSPF) 17, 18 incorporates a cost model in the importance distribution to generate more particles in high-risk regions of the statespace. Mathematically, the importance distribution is described as

$$
\begin{aligned}
q\left(\tilde{d}_{t}, \tilde{x}_{t} \mid \tilde{d}_{0: t-1}^{(i)}, x_{0: t-1}^{(i)}, y_{1: t}\right) & = \\
\gamma_{t} \cdot r\left(d_{t}\right) & p p\left(d_{t}, \tilde{x}_{t} \mid y_{1: t}\right)
\end{aligned}
$$

where $d_{t}$ is a set of discrete-valued states representing fault modes, $x_{t}$ is a set of continuous-valued states that describe the evolution of the system given those operating conditions, $r\left(d_{t}\right)$ is a positive risk function that is dependent on the fault mode, and $\gamma_{t}$ is a normalizing constant. It is critical to note that this $\mathrm{PF}$ approach makes use of exogenous models to evaluate and estimate the risk associated with every fault mode. This may prove to be difficult for implementation in some applications. This methodology was employed in FDI applications, improving the tracking of states that are critical to the performance of a sixwheel robot 18. However, this methodology has so far not been extended to prognostics

The RSPF-based approach for failure prognosis presented here ensures the existence of particles in the tails of the state pdf to represent the probability of events associated to high-risk conditions with low likelihood. In practice this implies a more conservative estimate of the remaining useful life (RUL) of a piece of equipment. The weights of the particles located at the 
tails of the pdf (which are updated each time a new measurement is obtained) represent an estimate of the mass probability of the tails, i.e., particles in the regions of the state space that are believed to have low likelihood; see Figure 1. In situations where the data show no signs of these events, the weights of these particles should decrease over time. Nevertheless, the information provided by these weights is of paramount importance, since it allows considering catastrophic events and highly non-monotonic phenomena in the schedule of the system operation and enables fast adjustments in prognosis results when incipient critical conditions are present 9-10. $\left\{\begin{array}{l}x_{1}(t+1)=x_{1}(t)+x_{2}(t) \cdot F(x(t), t, U)+\omega_{1}(t) \\ x_{2}(t+1)=x_{2}(t)+\omega_{2}(t)\end{array}\right.$
$y(t)=x_{1}(t)+v(t)$

where $x_{1}(t)$ is a state representing the fault dimension under analysis, $x_{2}(t)$ is a state associated with an unknown model parameter, $U$ are external inputs to the system (load profile, etc.), $F(x(t), t, U)$ is a general time-varying nonlinear function, and $\omega_{1}(t)$, $\omega_{2}(t), v(t)$ are white noises (not necessarily Gaussian). The nonlinear function $F(x(t), t, U)$ may represent a model, for example a model based on first principles, a neural network, or model based on fuzzy logic.

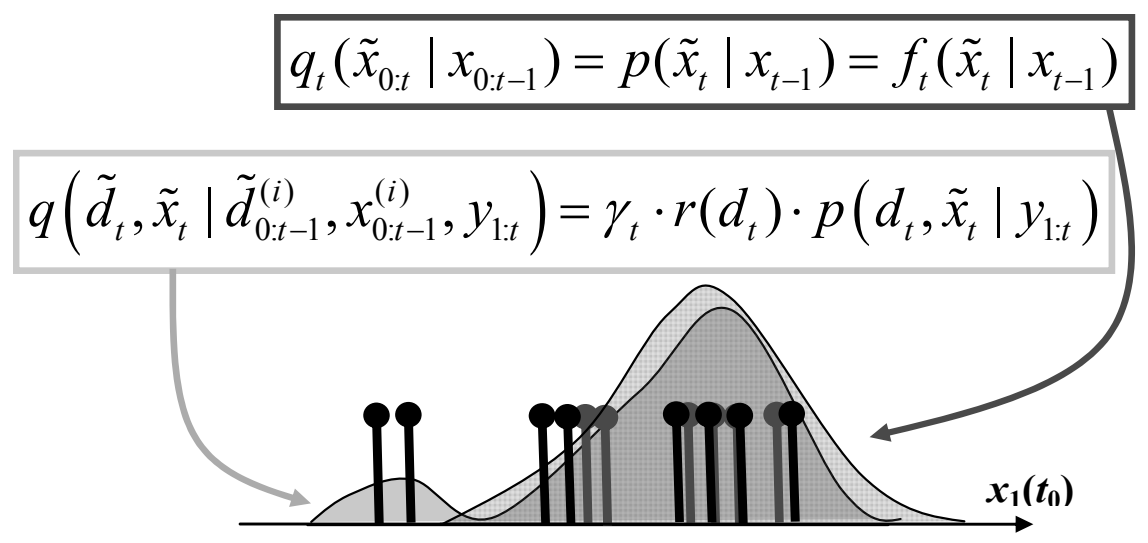

Figure 1. Particle population using either the a priori state pdf or a risk-sensitive pdf as importance distribution for $x_{1}(t)$

\subsection{PF-based failure prognosis}

Prognosis, and more generally, the generation of long-term prediction, is a problem that goes beyond the scope of filtering applications since it involves future time horizons. Hence, if PF-based algorithms are to be used for prognosis, a procedure is required that has the capability to project the current particle population into the future in the absence of new observations 7-8.

Any prognosis scheme requires the existence of at least one feature providing a measure of the severity of the fault condition under analysis (fault dimension). If many features are available, they can in principle be combined to generate a single signal. In Therefore, it is possible to describe the evolution in time of the fault dimension through the nonlinear state equation 6 :
By using the aforementioned state equation to represent the evolution of the fault dimension in time, one can generate long term predictions using kernel functions to reconstruct the estimate of the state pdf in future time instants 6-10:

$$
\begin{aligned}
\hat{p}\left(x_{t+k} \mid\right. & \left.\hat{x}_{1: t+k-1}\right) \approx \\
& \sum_{i=1}^{N} w_{t+k-1}^{(i)} K\left(x_{t+k}-E\left[x_{t+k}^{(i)} \mid \hat{x}_{t+k-1}^{(i)}\right]\right)
\end{aligned}
$$

where $K(\cdot)$ is a kernel density function, which may correspond to the process noise pdf, a Gaussian kernel or a rescaled version of the Epanechnikov kernel.

The resulting predicted state pdf contains critical information about the evolution of the fault dimension over time. One way to represent that information is through the expression of statistics (expectations, 95\% confidence intervals), either the End-ofLife (EOL) or the Remaining Useful Life 
(RUL) of the faulty system. A detailed procedure to obtain the RUL pdf from the predicted path of the state pdf is described and discussed in 7-8. Essentially, the RUL pdf can be computed from the function of probability-of-failure at future time instants. This probability is calculated using both the long-term predictions and empirical knowledge about critical conditions for the system. This empirical knowledge is usually incorporated in the form of thresholds for main fault indicators (also referred to as the hazard zones).

In real applications, hazard zones are expected to be statistically determined on the basis of historical failure data, defining a critical pdf with lower and upper bounds for the fault indicator ( $H_{l b}$ and $H_{u b}$, respectively). Let the hazard zone specify the probability of failure for a fixed value of the fault indicator, and the weights $\left\{w_{t+k}^{(i)}\right\}_{i=1 \ldots N}$ represent the predicted probability for the set of predicted paths, then the probability of failure at any future time instant (namely the RUL pdf) by applying the law of total probabilities, as shown in Eq. (5).

$$
\hat{p}_{\text {TTF }}(t)=\sum_{i=1}^{N} \operatorname{Pr}\left(\text { Failure } \mid X=\hat{x}_{t}^{(i)}, H_{l b}, H_{u b}\right) \cdot w_{t}^{(i)}
$$

Once the RUL pdf has been computed by combining the weights of predicted trajectories with the hazard zone specifications, prognosis confidence intervals, as well as the RUL expectation can be extracted.

\section{RSPF-based Prognostic Framework and Case Study Definition}

A RSPF-based approach for failure prognosis intends to represent the probability of rare events and highly non-monotonic phenomena within the structure of the stochastic nonlinear dynamic equation that describes the evolution of the fault condition. In particular, this approach proposes to use a variant of the RSPF algorithm (17-18), where the cost function (2) allows sampling particles $x^{(\mathrm{i})}(t)$ (i $\left.=1 \ldots N_{r}, N_{r}<<N\right)$ from regions of the state space representing high-risk conditions for the system and where the fault dimension has low likelihood (usually located in the tails of the pdf). The resulting particles are then used to generate a EOL estimate, following the framework that was presented in Section 2.2.

The aforementioned variant of the RSPF-based algorithm is implemented by modifying the kernel of the noise $\omega_{1}(t)$ in Eq. (3), and thus allowing some particles to be created in regions of the state space that represent extreme and rare changes in the evolution of the fault condition. In practice, this kernel modification implies a more conservative EOL estimate, and similarly for the RUL. The weights of the particles located at the tails of the original noise pdf represent an estimate of the mass probability of the tails and are updated accordingly to the new measurements. Information provided by these weights allows considering the probability of a catastrophic event and enables adjustments in prognosis results in the presence of incipient critical conditions.

As it has been previously mentioned, an arbitrary modification of the a priori state pdf has direct impact on the resulting RUL estimate for the faulty subsystem. We will present below an illustrative example to demonstrate the impact of considering sudden events on EOL calculation. The particular example focuses on energy storage devices. In this type of systems, continuous switching between charge and discharge cycles may cause momentary increments in the battery capacity (capacity regeneration), directly affecting its RUL.

The specific example describes the operation of a Li-Ion battery through two different operational profiles (charge and discharge) at room temperature. Charging was carried out in a constant current (CC) mode at 1.5[A] until the battery voltage reached $4.2[\mathrm{~V}]$ and then continued in a constant voltage mode until the charge current dropped to $20[\mathrm{~mA}]$. Discharge was carried out at a constant current (CC) level of 2[A] until the battery voltage fell to $2.5[\mathrm{~V}]$. Impedance measurements provide insight into the internal battery parameters that change as aging progresses. Repeated charge and discharge cycles result in aging of the batteries. Impedance measurements were done through an electrochemical impedance spectroscopy (EIS) frequency sweep from 
$0.1[\mathrm{~Hz}]$ to $5[\mathrm{kHz}]$. The experiments were stopped when the batteries reached end-oflife (EOL) criteria, which was a $40 \%$ fade in rated capacity (from 2[A-hr] to 1.2[A-hr]). This dataset can be used both for the prediction of both remaining charge (for a given discharge cycle) and remaining useful life (RUL).

Instead of a physics-based model we will employ here a population-growth-based model 6 that has been trained using online capacity measurements (fault dimension in [A-hr]):

$$
\begin{aligned}
& \left\{\begin{array}{l}
x_{1}(t+1)=x_{1}(t)+C \cdot x_{2}(t) \cdot\left(a-b \cdot t+t^{2}\right)^{m}+\omega_{1}(t) \\
x_{2}(t+1)=x_{2}(t)+\omega_{2}(t) \\
x_{3}(t+1)=\alpha \cdot x_{3}(t)+\omega_{3}(t)
\end{array}\right. \\
& y(t)=x_{1}(t)+x_{3}(t)+v(t)
\end{aligned}
$$

where $x_{1}(t)$ is a state representing the fault dimension, $x_{2}(t)$ is a state associated with an unknown model parameter, $a, b, C$ and $m$ are constants associated to the duration and intensity of the battery load cycle (external input $U$ ), and $0 \leq \alpha \leq 1$ is a parameter that characterizes the capacity regeneration phenomena.

The objective of a prognostic routine applied to the system defined by (6), and particularly for the ones based on PF algorithms, is to estimate (preferably in an online fashion) the current battery capacity, isolating the effect of the regeneration phenomena, and to use that information to estimate the amount of cycles remaining until this quantity falls below the threshold of 1.2[A-hr].

As it has been previously described, the implementation of a RSPF-based algorithm differs from classic PF-based approaches in the fact that it modifies the noise kernel $\omega_{1}(t)$, allowing to sample from regions of the state space associated to events that could imply strong (or rapid) changes in the current RUL estimate (by direct modification of the importance probability distribution, 13-14). Particularly, this case study defines the noise kernel $\omega_{1}(t)$ as independent noise distributing as a Gaussian mixture; that is, the distribution of the process noise $\omega_{1}(t)$ can be written as:

$$
\omega_{1}(t) \sim \delta \cdot \omega_{1}^{\prime}(t)+(1-\delta) \cdot \omega_{1}^{*}(t)
$$

where $\omega_{1}^{*}(t) \sim N\left(d, \sigma^{* 2}\right), \quad d=E\left\{\omega_{1}^{*}(t)\right\} \neq 0$, $\omega_{1}^{\prime}(t) \sim N\left(0, \sigma^{\prime 2}\right)$, and $0 \leq \delta \leq 1$.

Consequently, RSPF-based prognostic algorithms have three extra parameters to be defined, when compared with classic PFbased implementations. However, if the variances of the Gaussian kernels are selected such that $\sigma^{\prime}=\sigma^{*}$, and both of them are equal to the process noise variance of the classic $\mathrm{PF}$ approach, then only $d$ and $\delta$ must be considered as extra design parameters.

In the test case used for the analysis, feature data associated to the fault is fed into the RSPF-based prognostic algorithm to estimate the EOL pdf. Arbitrary initial conditions are set for the unknown model parameter in Eq. (6), and it is known that failure mechanisms may undergo changes. The analysis will focus on the quality of the estimate for the state components $x_{1}$ and $x_{3}$, after each capacity regeneration phenomena and on the accuracy exhibited by the corresponding EOL pdf estimate. It is expected that RSPF-based routines will anticipate possible sudden changes in the system's operating condition (such as changes from discharge to charge) helps to adjust states estimates in a rapid manner, therefore improving its capability to predict future behaviors. Performance comparison is done with respect to a classic (SIR) PF-based prognostic framework 7-8, given same initial conditions. It should be noted that the implementation chose here considers a correction loop that simultaneously updates the variance of kernel associated to the white noise $\omega_{2}(t)$ according to the short-term prediction 6,9 .

Three qualitative aspects are considered in the design of the RSPF-based prognosis framework: (1) Effect of parameter $d$ in online state estimates, (2) effect of parameter $\delta$ in the aforementioned estimates, and (3) effect of parameter $d$ in EOL pdf estimates. Guidelines suggested in 10 have been used to select adequate values for the aforesaid parameters.

The implementation of the scheme presented in this section has been performed using MATLAB ${ }^{\circledR}$ environment. A complete description of the results obtained when implementing the suggested RSPF-based prognosis approach for RUL estimation in the 
case study, and a comparison with classic PFbased routines, follows in Section 4.

\section{RSPF-based Prognostic Framework: Assessment and Evaluation Using Performance Metrics}

Estimates obtained from a Particle Filtering algorithm are based on the realization of a stochastic process and measurement data. Assessment or comparison between different strategies should consider performance statistics rather than a performance assessment based on a single experiment or realization. For that reason, all results presented in this paper consider the statistical mean of 40 realizations for the particle filter algorithm and a single measurement data set.

In addition, the assessment and evaluation of prognostic algorithms require appropriate performance metrics capable of incorporating concepts such as "accuracy" and "precision" of the RUL pdf estimate 19. "Accuracy" is defined as the difference between the actual failure time and the estimate of its expectation, while "precision" is an expression of the spread (e.g., standard deviation). These indicators should also consider the fact that both the $R U L$ and $E_{\mathrm{t}}\{R U L\}$ (estimate, at time $t$, of the expectation of the equipment RUL) are random variables. Moreover, it is desirable that all indicators assume that, at any time $t$, it is possible to compute an estimate of the $95 \%$ confidence interval $\left(C I_{\mathrm{t}}\right)$ for the EOL.

In particular this paper uses three indicators to evaluate prognostic results, which are presented and detailed in 9: (1) RUL precision index (RUL-OPI), (2) RUL accuracy-precision index, and (3) RUL online steadiness index (RUL-OSI). RULOPI considers the relative length of the 95\% confidence interval computed at time $t$ $\left(\mathrm{CI}_{t}\right)$, when compared to the RUL estimate. It is expected that the more data the algorithm processes, the more precise the prognostic becomes:

$$
\begin{aligned}
& \left.\operatorname{RUL-OPI}(t)=e^{-\left(\frac{\sup \left(C I_{t}\right)-\inf \left(C I_{t}\right)}{E_{t}\{E O L\}}\right)-t}\right) \\
& 0<\operatorname{RUL-OPI}(t) \leq 1, \forall t \in\left[1, E_{t}\{E O L\}\right), t \in \mathbb{N} .
\end{aligned}
$$

The RUL accuracy-precision index, measures the error in EOL estimates relative to the width of its $95 \%$ confidence interval $\left(\mathrm{CI}_{t}\right)$. It also penalizes late predictions, i.e., whenever the expected EOL $E_{t}\{\mathrm{EOL}\}>$ GroundTruth $\{\mathrm{EOL}\}$ (actual failure happened before the expected time). This indicator can be computed only after the end of the simulation.

Finally, the RUL-OSI considers the variance of the EOL conditional expectation, computed with measurement data available at time $t$. Good prognostic results are associated to small values for the RUL-OSI. All performance metrics will be evaluated at all time instants.

In the case study presented in this paper (RUL/EOL estimation of a Li-Ion battery) the time is measured in cycles of operation. A cycle of operation consists of two different operational states applied to the battery at room temperature (charge and discharge).

It is essential to note that algorithm assessment (both for the classic PF and RSPF-based prognosis frameworks) only considered RUL estimates generated until the $120^{\text {th }}$ cycle of operation, which corresponded to about $75 \%$ of the actual useful life of the battery (actual EOL of the experiment is 159 cycles), since it is of more interest to evaluate the algorithm's performance when the size of the prediction window is large enough to allow for corrective actions. Moreover, given that PF-based prognostic algorithms tend to improve their performance as the amount of available data increases 14-16, the closer the system is to the actual EOL, the more accurate the resulting EOL estimate. This needs to be kept in mind when analyzing results presented both in Figure 2 and Figure 3.

Figure 2 (a) shows online tracking for the battery capacity (coarse trace) using a classic PF-based prognostic approach until the $120^{\text {th }}$ cycle of operation, the hazard zone around 1.2 [A-hr] (marked as a horizontal band), and the $95 \%$ confidence interval of EOL (coarse vertical dashed lines) computed at the $120^{\text {th }}$ cycle. Figure 2 (b) only shows the EOL pdf estimate computed at the end of the $120^{\text {th }}$ cycle of operation. The result of the classic PF-based prognostic approach is accurate to two cycles (the expected value of the EOL pdf is 161 cycles, while the ground truth data 
for the EOL is 159 cycles). However, the state estimate, in this case, does not exhibit the same level of accuracy when describing capacity regeneration phenomena registered at the $19^{\text {th }}, 30^{\text {th }}$, and $47^{\text {th }}$ cycles of operation.; see Table 1. In fact, regeneration phenomena momentarily affect the algorithm performance, in particular in terms of steadiness of the solution 9 as the analysis based on performance metrics will corroborate shortly.
Table 1. Estimates for system output $y(t)$

\begin{tabular}{llll}
\hline \multirow{2}{*}{ Cycle } & $\begin{array}{l}\text { Measured } \\
\text { data }\end{array}$ & $\begin{array}{l}E_{t}\{y(t)\} \\
\text { Classic PF-based } \\
\text { routine }\end{array}$ & $\begin{array}{l}E_{t}\{y(t)\} \\
\text { RSPF-based } \\
\text { routine }\end{array}$ \\
$19^{\text {th }}$ & 1.98 & 1.77 & 1.80 \\
$30^{\text {th }}$ & 1.92 & 1.70 & 1.80 \\
$47^{\text {th }}$ & 1.82 & 1.62 & 1.67 \\
\hline \hline
\end{tabular}
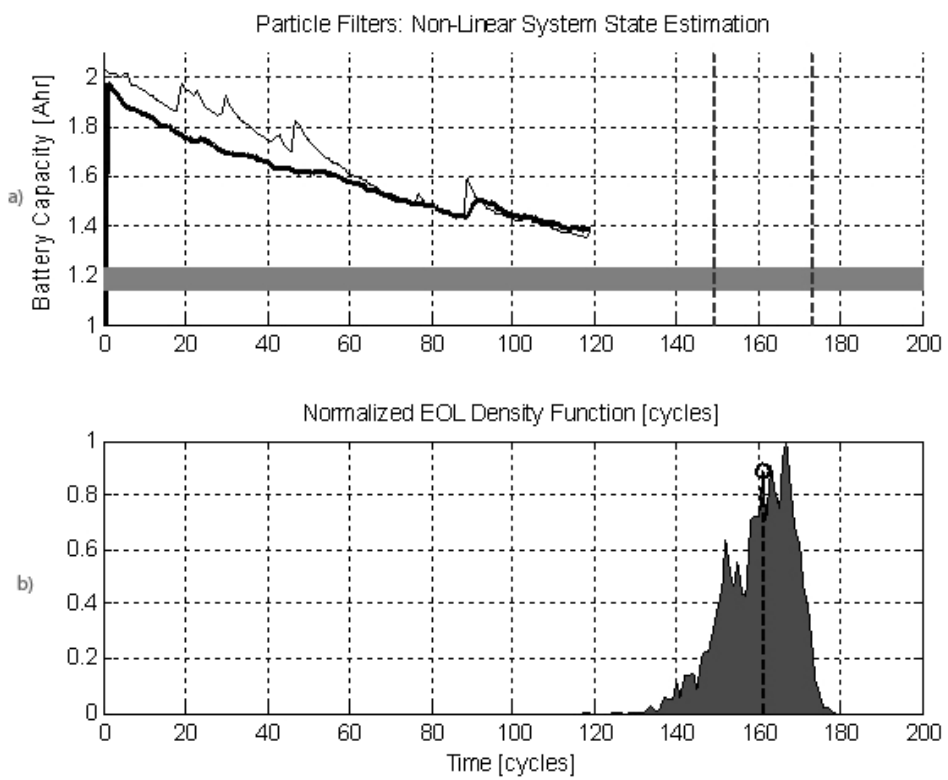

Figure 2. Case study. (a) Measurement data (fine trace), PF-based estimate (coarse trace), and 95\% confidence interval (vertical read dashed lines). (b) EOL pdf estimate using classic PF-based prognosis framework and its expectation
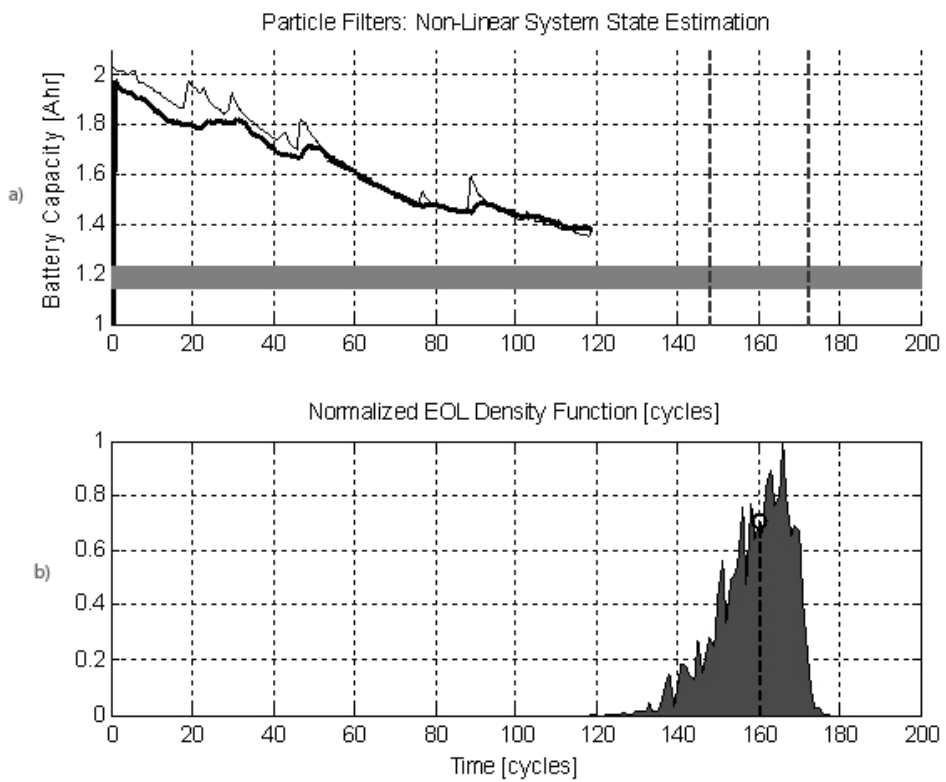

Figure 3. (a) Measurement data (fine trace), PF-based estimate (coarse trace), and $95 \%$ confidence interval. (b) EOL pdf estimate using RSPF-based prognosis framework and its expectation 
One can overcome these difficulties to some degree using the RSPF-based prognosis framework. We implemented the RSPF as outlined in Section 3, where the risk noise kernel $\omega_{1}^{*}(t) \sim N\left(0.04,1.25 \cdot 10^{-4}\right) \quad$ and $\delta=0.95$ are used to build the process noise kernel $\omega_{1}(t)$ of the first component of the state vector in model equation (6). Figure 3 (a) shows online tracking for the battery capacity (coarse trace) using the aforesaid RSPF-based prognostic approach until the $120^{\text {th }}$ cycle of operation, the hazard zone around 1.2 [A-hr], and the $95 \%$ confidence interval of EOL computed at the $120^{\text {th }}$ cycle. Figure 3 (b) shows the EOL pdf estimate computed at the end of the $120^{\text {th }}$ cycle of operation.

Figure 3 shows that the RSPF-based prognostic approach is equally capable of providing an accurate estimate of the battery RUL with an expected value of the EOL pdf (computed at the $120^{\text {th }}$ cycle of operation) of 160 cycles, while the ground truth data for the EOL is 159 cycles). However, it is more interesting to note that the RSPF noticeably improves the state estimate at early stages of the test, particularly between the $20^{\text {th }}$ and the $60^{\text {th }}$ cycle of operation (see Table 1), allowing a better description of the regeneration phenomena that affect the Li-Ion kernel $\left.\omega_{1}^{*}(t)\right)$ can help to improve the state estimate when rare, unlikely events or highly non-monotonic phenomena occur.

Even more compelling, similar conclusions can be drawn when using prognostic performance metrics to assess the performance of the plain vanilla PF and the RSPF-based approaches; Figure 4 summarizes tracking and prediction with all tracking estimates generated until the $120^{\text {th }}$ cycle.

Figure 4 (a) shows the evaluation of RULOPI as measurement data are included in a sequential manner into the prediction algorithm. One of the main characteristics of this indicator is that it penalizes the width of the $95^{\text {th }} \%$ confidence interval as the system approaches EOL. The value of this indicator is comparable for both algorithms (around 0.5 near the end of the experiment) More important than the absolute value of the indicator is the fact that in the performance (as expressed by RUL-OPI) the RSPF-based prognostic routine does not suffer in comparison to the classic approach, even though the prior density is modified at each time instant to include particles in areas of the state space with low likelihood (according to measurements).
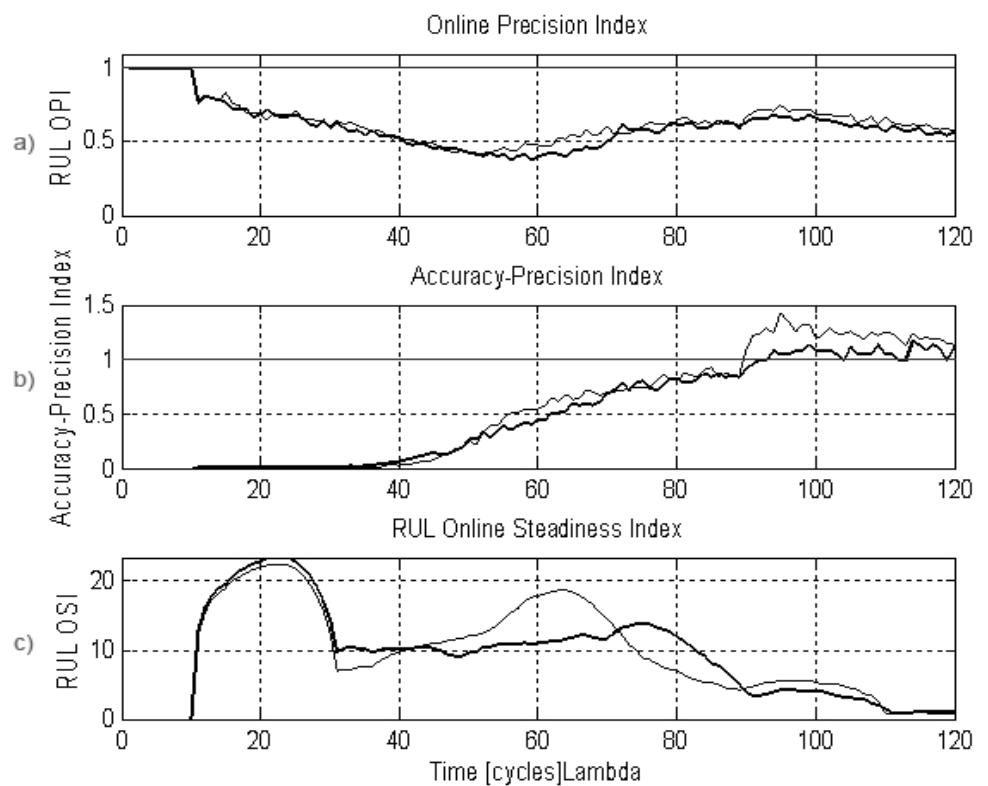

Figure 4. Performance metric evaluation in case study. Comparison between classic PF (fine trace) and RSPF-based prognosis framework (coarse trace)

battery. This demonstrates how the existence of particles in areas of low likelihood (as a direct consequence of the use of the risk
However, both the accuracy-precision and the RUL-OSI indices indicate noticeable advantages of the RSPF-based prognostic 
framework when compared to its classic version as illustrated in Figure 4 (b) and Figure 4 (c). The evaluation of the accuracyprecision index clearly shows improved performance in the case of RSPF-based algorithm, which translates into better estimates for the EOL conditional expectation. Similar conclusions can be obtained from Figure 4 (c), where the steadiness RUL-OSI index shows smaller values for extended periods of time (and thus better performance) for the RSPF-based algorithm.

Previous research work 5-12 has already shown better results when using classic PFbased prognostic framework, compared to other approaches. For this reason, this performance analysis did not consider other methods such as the extended Kalman filter in its formulation.

\section{Conclusions}

This paper presents a case study where a RSPF-based prognosis framework has been applied to estimate the remaining useful life of an energy storage device (Li-Ion battery). A comparison based on prognosis performance metrics indicates that the RSPFbased prognostic approach is more suitable than classic PF methods to represent highly non-monotonic phenomena such as capacity regeneration phenomena between charging periods, in terms of accuracy of the state estimate and steadiness of the RUL estimate. We surmise that the existence of particles in the tails of the state pdf allows the RSPFbased prognostic algorithm to generally provide a more conservative estimate of the RUL of the faulty piece of equipment. We surmise that it also helps to incorporate the probability of rare and costly events in the evolution of the fault condition in time.

\section{Acknowledgements}

The authors would like to acknowledge the support of NASA Aviation Safety Program/IVHM Project NRA NNA08BC20C, Thanks also go to Dr. Johan Reimann and Dr. Gregory Kacprzynski of Impact Technologies for valuable discussions.

\section{REFERENCES}

1. SHAFER, G., A Mathematical Theory of Evidence, Princeton, N.J: Princeton University Press, 1976.

2. KHIRIPET, N., G. VACHTSEVANOS, A. THAKKER, T. GALIE, A New Confidence Prediction Neural Network for Machine Failure Prognosis, Proceedings of Intelligent Ships Symposium IV, Philadelphia, PA, April 2-3, 2001.

3. SPECHT, D. F., A General Regression Neural Network, IEEE Trans on Neural Networks, vol. 2, no. 6, November, 1991, pp. 568-76.

4. CRUSE, T. A., Probabilistic Systems Modeling and Validation, HCF 2004, March 16-18, 2004.

5. ORCHARD, M., B. WU, G. VACHTSEVANOS, A Particle Filter Framework for Failure Prognosis, Proceedings of World Tribology Congress III, Washington DC, Sept. 1216, 2005.

6. ORCHARD, M., G. KACPRZYNSKI, K. GOEBEL, B. SAHA, G. VACHTSEVANOS, Advances in Uncertainty Representation and Management for Particle Filtering Applied to Prognostics, 2008 International Conference on Prognostics and Health Management PHM 2008, Denver, CO, USA, October 9 - 12, 2008.

7. ORCHARD, M., On-line Fault Diagnosis and Failure Prognosis Using Particle Filters. Theoretical Framework and Case Studies, Publisher: VDM Verlag Dr. Müller Aktiengesellschaft \& Co. KG, Saarbrücken, Germany, April 2009, 108 pages. Atlanta: The Georgia Institute of Technology, Diss., 2007.

8. ORCHARD, M. G. VACHTSEVANOS, A Particle Filtering Approach for OnLine Fault Diagnosis and Failure Prognosis, Transactions of the Institute of Measurement and Control, vol. 31, no. 3-4, June 2009, pp. 221-246.

9. ORCHARD, M., F. TOBAR, G. VACHTSEVANOS, Outer Feedback Correction Loops in Particle Filtering- 
based Prognostic Algorithms: Statistical Performance Comparison, Studies in Informatics and Control, vol.18(4), December 2009, pp. 295-304.

10. ORCHARD, M., L. TANG, K. GOEBEL, G. VACHTSEVANOS, A Novel RSPF Approach to Prediction of High-Risk, Low-Probability Failure Events, First Annual Conference of the Prognostics and Health Management Society, 2009, San Diego, CA, USA.

11. PATRICK, R., M. ORCHARD, B. ZHANG, M. KOELEMAY, G. KACPRZYNSKI, A. FERRI, G. VACHTSEVANOS, An Integrated Approach to Helicopter Planetary Gear Fault Diagnosis and Failure Prognosis, $42^{\text {nd }}$ Annual Systems Readiness Technology Conference, AUTOTESTCON 2007, Baltimore, USA, September 2007.

12. ZHANG, B., T. KHAWAJA, R. PATRICK, M. ORCHARD, A. SAXENA, G. VACHTSEVANOS, A Novel Blind Deconvolution De-Noising Scheme in Failure Prognosis, IEEE Transactions on Instrumentation and Measurement, vol. 58, no. 2, February 2009, pp. 303-310.

13. ANDRIEU, C., A. DOUCET, E. PUNSKAYA, Sequential Monte Carlo Methods for Optimal Filtering, in Sequential Monte Carlo Methods in Practice, A. Doucet, N. de Freitas, and N. Gordon, Eds. NY: Springer-Verlag, 2001.
14. ARULAMPALAM, M. S., S. MASKELL, N. GORDON, T. CLAPP, A Tutorial on Particle Filters for Online Nonlinear/Non-Gaussian Bayesian Tracking, IEEE Transactions on Signal Processing, vol. 50, no. 2, Feb. 2002, pp. $174-188$.

15. DOUCET, A., On Sequential Monte Carlo Methods for Bayesian Filtering, Technical Report, Engineering Department, University of Cambridge, UK, 1998.

16. DOUCET, A., N. DE FREITAS, N. GORDON, An introduction to Sequential Monte Carlo methods, in Sequential Monte Carlo Methods in Practice, A. Doucet, N. de Freitas, and N. Gordon, Eds. NY: Springer-Verlag, 2001.

17. THRUN, S., J. LANGFORD, V. VERMA, Risk Sensitive Particle Filters, Neural Information Processing Systems (NIPS), Dec. 2001.

18. VERMA, V., G. GORDON, R. SIMMONS, S. THRUN, Particle Filters for Rover Fault Diagnosis, IEEE Robotics \& Automation Magazine, pp. 56 - 64, June 2004.

19. VACHTSEVANOS, G., F.L. LEWIS, M.J. ROEMER, A. HESS, B. WU, Intelligent Fault Diagnosis and Prognosis for Engineering Systems, Hoboken, NJ, John Wiley and Sons, 2006. 